\title{
SYSTEMS OF CONTINUOUS AND DISCONTINUOUS SIMPLE GROUPS.
}

BY DR. LEONARD E. DICKSON.

(Read at the April Meeting of the Society, 1897.)

$\S 1$.

Known systems of discontinuous simple groups.

The following list gives the orders of the systems of discontinuous simple groups which have thus far been determined. Here $p=$ prime, $m$ and $n=$ integers.

$$
\begin{gathered}
p \\
\frac{1}{2} n ! \quad(n>4) \\
\frac{\left(p^{n m}-1\right)\left(p^{n m}-p^{n}\right) \cdots\left(p^{n m}-p^{n m-n}\right)}{d\left(p^{n}-1\right)}
\end{gathered}
$$

$(m, n, p)+(2,1,2)$ or $(2,1,3) ; d$ is the greatest common divisor of $m$ and $p^{n}-1$.

$$
\begin{aligned}
& \frac{1}{2}\left(p^{2 n m}-1\right) p^{n(2 m-1)}\left(p^{n(2 m-2)}-1\right) p^{n(2 m-3)} \cdots\left(p^{2 n}-1\right) p^{n} \\
& \text { (for } p>2 \text { ) } \\
& \left(2^{2 n m}-1\right) 2^{n(2 m-1)} \cdots \cdots\left(2^{2 n}-1\right) 2^{n} \quad(m>2) \text {. } \\
& \text { (5) }\left(2^{m}-1\right) \cdot\left(2^{2 m-2}-1\right) 2^{2 m-2}\left(2^{2 m-4}-1\right) 2^{2 m-4} \cdots\left(2^{2}-1\right) 2^{2} \\
& (m>2) \text {. } \\
& \left(2^{m}+1\right) \cdot\left(2^{2 m-2}-1\right) 2^{2 m-2} \cdots\left(2^{2}-1\right) 2^{2} \quad(m>2) .
\end{aligned}
$$

The two triply-infinite systems (3) and (4) were obtained* by generalizing to the Galois Field of order $p^{n}$ the two doubly-infinite systems set up by Jordan. $\dagger$ The system (3) is abtained in the decomposition of the general linear homogeneous group on $m$ indices, in whose substitutions both indices and coefficients are " marks" of the Galois Field of order $p^{n}$. Concretely, (3) is the group of

* 3. L. E. Dickson. "The analytic representation of substitutions on a power of a prime number of letters, with a discussion of the linear group.-Annals of Mathematics, 1897.

4. L. E. Dickson. A triply-infinite system of simple groups.-Quarterly Journal of Mathematics, vol. 29, 1897.

These results were announced in a paper read before the AMERICAN Mathematical Society at Buffalo, August 31, 1896.

† Traité des Substitutions, p. 106 and pp. 176, 178. 
linear fractional substitutions of determinant unity on $m-1$ indices. The system (4) is obtained in the decomposition of the Abelian group on $2 \mathrm{~m}$ indices; viz, that subgroup of the general linear homogeneous group on $2 m$ indices (in which both coefficients and indices are marks of the Galois Field of order $p^{n}$ ), every substitution of which if operating simultaneously on two such sets of $2 \mathrm{~m}$ indices

$$
\xi_{i}, \eta_{i} ; \rho_{i}, \sigma_{i} \quad(i=1 \cdots m)
$$

multiplies the function

by a constant factor.

$$
\sum_{i=1}^{m}\left(\xi_{i} \sigma_{i}-\eta_{i} \rho_{i}\right)
$$

The systems (3) and (4) have in common the doublyinfinite system (viz, (3) for $m=2$ and (4) for $m=1$ ) set up first by Professor E. H. Moore* and a few months later by Professor W. Burnside $\dagger$ as a generalization of the well known modular group of order

$$
\frac{1}{2} p\left(p^{2}-1\right), \quad(p>3) \text {. }
$$

The system (5) is due to Jordan, ibid, p. 205. It is obtained in the decomposition of the First-Hypoabelian group. For the sake of comparison I have changed the form of the expression giving its order, viz :

$$
\begin{aligned}
& \left(P_{m}-1\right) 2^{2 m-2}\left(P_{m-1}-1\right) 2^{2 m-4} \cdots\left(P_{2}-1\right) 2^{2} \quad(m>2) \\
& \text { where } \\
& P_{s}=2^{2 s-1}+2^{s-1} \text {. }
\end{aligned}
$$

The system (6) is obtained in the decomposition of the Second-Hypoabelian group. The order of the latter is given incorrectly in Jordan, ibid, p. 207, as equal to the order of the First-Hypoabelian group. The number of solutions of the congruence (42), p. 207, is $P_{n}$ and not $2^{2 n}-P_{n}$; the reference in $\S 282$ should be to $\$ 259$ and not to $\$ 260$. I have not seen the simple groups (6) given explicitly anywhere.

Recentlył I have succeeded in generalizing the system (5) to a doubly infinite system, the expression for the order being rather complicated. The Second-Hypoabelian

* Abstract in the Bulletin of The Mathematical Society, December, 1893 ; complete in the Mathematical Papers of the Chicago Congress.

$\dagger$ "On a Class of Groups defined by Congruences," Proceedings of the London Mathematical Society, vol. 25, pp. 113-139. February, 1894.

$\ddagger$ "The First-Hypoabelian group generalized," offered April 1st to the Quarterly Journal of Mathematics. 
group presents serious difficulties to generalization, as the conditions defining it (Jordan, $\$ 277$ ) do not lead to a group at all in the $G F\left[p^{n}\right], n>1$.

\section{$\S 2$.}

Systems of finite continuous transformation groups which are simple.*

Through the work of Killing $\dagger$, which has been carried out with more rigor by Cartan, \$ we know that all continuous finite simple transformation groups, aside from five isolated ones, belong to one of the four systems set up by Sophus Lie, each of such fundamental importance in geometry and analysis :

(a) The groups with $l(l+2)$ parameters, isomorphic (= gleichzusammengesetzt) with the general projective group of $R_{l}$ (= space of $l$ dimensions).

$\left(b_{1}\right)$ The groups with $l(2 l+1)$ parameters, isomorphic with the general projective group of a non-degenerate surface of the second order in $R_{2 l}$ and hence also with the largest group of conform transformations in $R_{2 l-1}$.

$\left(b_{2}\right)$ The groups with $l(2 l-1)$ parameters, isomorphic with the general projective group of a non-degenerate surface of the second order in $R_{2 l-1}$. (Here must $l>2$.)

(c) The groups with $l(2 l+1)$ parameters, isomorphic with the general projective group of a linear complex in $R_{2 l-1}$.

The five isolated simple groups not falling in these four classes contain respectively $14,52,78,133$ and 248 parameters and exist as point-transformation groups in respectively 5, 15, 16, 27 and 57 variables (but no fewer) ; however, as Berührungstransformation groups in fewer variables.

$$
\$ 3 .
$$

Elementary deduction of the groups (c) and proof of their simplicity.

The following elementary proof had its origin in an attempt to carry over into Lie's continuous group theory the

\footnotetext{
* Professor LIE has determined four classes of infinite transformation groups which are simple; e. g., all transformations of space of $n$ dimensions ; again, all Berührungstransformationen.

† Wilmelm KILLING, "Die Zusammensetzung der stetigen endlichen Transformationsgruppen, Math. Ann., vols. 31, 33, 34, 36 (particularly vol. 33).

†ÉLIE CARTaN, "Ueber die einfachen Transformationsgruppen," Leipziger Berichte, pp. 393-420, 1893 ; "Sur la structure des groupes simples finis et continus," Comptes Rendus, vol. 116, p. 784 (17 Apr., 1893 ) and p. 962 ; also Thèse, Paris, Nony., 1894.
} 
investigations of Jordan on Abelian groups (whose decomposition led to the system of simple groups (4)). It is remarkable how much simpler and more elegant the problem becomes for continuous groups. That the simple groups thus obtained are identical with the groups (c) is proved below.

Of the transformations of the general linear homogeneous group on $2 n$ variables, we consider those which, when operating simultaneously on two independent sets of variables

$$
x_{i}, y_{i} ; \quad \xi_{i}, \eta_{i} \quad(i=1,2, \cdots n)
$$

leave invariant the function

$$
\varphi=\sum_{i=1}^{n}\left(x_{i} \eta_{i}-\xi_{i} y_{i}\right) \text {. }
$$

Let $G$ denote the sub-group of the linear homogeneous group thus defined and $S$ an arbitrary transformation of $G$; viz.:

$$
\begin{aligned}
& x_{i}^{\prime}=\sum_{j=1}^{n}\left(a_{j}^{(i)} x_{j}+c_{j}^{(i)} y_{j}\right) \\
& y_{i}^{\prime}=\sum_{j=1}^{n}\left(b_{j}^{(i)} x_{j}+d_{j}^{(i)} y_{j}\right)
\end{aligned} \quad(i=1 \cdots n)
$$

The conditions that $S$ shall leave $\varphi$ invariant are :

$$
\begin{aligned}
\sum_{\nu=1}^{n}\left(a_{\mu}^{(\nu)} d_{\mu}^{(\nu)}-b_{\mu}^{(\nu)} c_{\mu}^{(\nu)}\right) & =1, \quad \sum_{\nu=1}^{n}\left(a_{\mu}^{(\nu)} d_{\lambda}^{(\nu)}-b_{\mu}^{(\nu)} c_{\lambda}^{(\nu)}\right)=0, \\
\sum_{\nu=1}^{n}\left(a_{\mu}^{(\nu)} b_{\lambda}^{(\nu)}-b_{\mu}^{(\nu)} a_{\lambda}^{(\nu)}\right) & =0, \quad \sum_{\nu=1}^{n}\left(c_{\mu}^{(\nu)} d_{\lambda}^{(\nu)}-d_{\mu}^{(\nu)} c_{\lambda}^{(\nu)}\right)=0, \\
(\mu, \lambda & =1 \cdots n, \mu \neq \lambda),
\end{aligned}
$$

altogether

$$
n+n(n-1)+\frac{n(n-1)}{2}+\frac{n(n-1)}{2}=n(2 n-1)
$$

conditions.

Now $S$ reduces to the identity if

$$
\begin{gathered}
a_{i}^{(i)}=1, d_{i}^{(i)}=1, \quad a_{j}^{(i)}=0, d_{j}^{(i)}=0 \quad(i, j=1 \cdots n, i \neq j) \\
c_{j}^{(i)}=0, b_{j}^{(i)}=0 \quad(i, j=1 \cdots n) .
\end{gathered}
$$

Hence we reach the general infinitesimal transformation of $G$ by writing

$$
a_{i}^{(i)}=1+\alpha_{i}^{(i)} \delta t, d_{i}^{(i)}=1+\delta_{i}^{(i)} \delta t, \alpha_{j}^{(i)}=\alpha_{j}^{(i)} \delta t, \text { etc. }
$$


and subjecting the $\alpha_{j}^{(i)}, \beta_{j}^{(i)}, \gamma_{j}^{(i)}, \delta_{j}^{(i)}$ to the conditions following from the above $n(2 n-1)$; viz,

$$
\begin{gathered}
\alpha_{\mu}^{(\mu)}+\delta_{\mu}^{(\mu)}=0, \quad \delta_{\lambda}^{(\mu)}+\alpha_{\mu}^{(\lambda)}=0 \\
\beta_{\lambda}^{(\mu)}-\beta_{\mu}^{(\lambda)}=0, \quad \gamma_{\mu}^{(\lambda)}-\gamma_{\lambda}^{(\mu)}=0 \\
(\mu, \lambda=1 \cdot n, \mu \neq \lambda) .
\end{gathered}
$$

The most general infinitesimal transformation of the group $G$ is thus

$$
\begin{gathered}
\delta x_{i} \equiv x_{i}^{\prime}-x_{i}=\sum_{j=1}^{n}\left(\alpha_{j}^{(i)} x_{j}+\gamma_{j}^{(i)} y_{j}\right) \\
\delta y_{i} \equiv y_{i}^{\prime}-y_{i}=\sum_{j=1}^{n}\left(\beta_{j}^{(i)} x_{j}-\alpha_{i}^{(j)} y_{j}\right)
\end{gathered}
$$

where $\quad \beta_{j}^{(i)}=\beta_{i}^{(j)}, \gamma_{j}^{(i)}=\gamma_{i}^{(j)} \quad(i, j=1 \cdots n, i \neq j)$.

Putting in turn each of the $2 n^{2}+n$ independent constants remaining equal 1 and all the others zero, we reach the $2 n^{2}+n$ linearly independent transformations of $G$ :

$$
\begin{gathered}
Q_{i j} \equiv x_{j} \frac{\partial^{\prime} f}{\partial x_{i}}-y_{i} \frac{\partial f}{\partial y_{j}} \quad(i, j=1 \cdots n) . \\
L_{i} \equiv y_{i} \frac{\partial f}{\partial x_{i}} ; \quad L_{i}^{\prime} \equiv x_{i} \frac{\partial f}{\partial y_{i}} \quad(i=1 \cdots n) . \\
R_{i j} \equiv x_{i} \frac{\partial f}{\partial y_{j}}+x_{j} \frac{\partial f}{\partial y_{i}} ; \quad N_{i j} \equiv y_{i} \frac{\partial f}{\partial x_{j}}+y_{j} \frac{\partial f}{\partial x_{i}} \quad(i, j=1 \cdots n, i \neq j) .
\end{gathered}
$$

The notation $Q_{i j}, L_{i}$, etc., corresponds to that of Jordan, p. 174.

We verify the following Klammerausdruck relations :

$$
\begin{gathered}
\left(L_{i} Q_{i i}\right)=2 L_{i} ;\left(L_{i} Q_{i j}\right)=0 ;\left(L_{i} Q_{j i}\right)=N_{i j^{\circ}} \\
\left(L_{i} L_{i}^{\prime}\right)=-Q_{i i} ;\left(L_{i} R_{i j}\right)=-Q_{i j} ;\left(L_{i} N_{i j}\right)=0 . \\
\left(L_{i}^{\prime} Q_{i i}\right)=-2 L_{i}^{\prime} ;\left(L_{i}^{\prime} Q_{i j}\right)=-R_{i j} ;\left(L_{i}^{\prime} Q_{j i}\right)=0 . \\
\left(L_{i}^{\prime} N_{i j}\right)=Q_{j i} ;\left(L_{i}^{\prime} R_{i j}\right)=0 . \\
\left(R_{i j} Q_{i i}\right)=-R_{i j} ;\left(R_{i j} Q_{j}\right)=-2 L_{j}^{\prime} ;\left(R_{i j} N_{i j}\right)=Q_{i i}+Q_{j j^{*}} \\
\left(N_{i j}, Q_{i i}\right)=N_{i j} ;\left(N_{i j} Q_{i j}\right)=2 L_{i} ;\left(N_{i j} Q_{k j}\right)=N_{i k} .
\end{gathered}
$$

Lemma: If an invariant subgroup $H$ of $A$ contains the single infinitesimal transformation $L_{i}$ ( $i=$ fixed), it contains all the transformations of $G$.

If $H$ contains $L_{i}$, it contains $N_{i j}(j=1 \cdots n, j \neq i)$, 
since

$$
\left(L_{i} Q_{j i}\right)=N_{i v^{*}}
$$

But

$$
\left(N_{i j} Q_{j i}\right)=2 L_{j}
$$

Hence $H$ would contain every $L_{i}$ and $N_{i j}$; hence also

$$
\begin{gathered}
\left(L_{i} R_{i j}\right)=-Q_{i j} \quad(j \neq i) ;\left(L_{i}^{\prime} \mathcal{L}_{i}\right)=Q_{i i} \\
\left(Q_{i i} L_{i}^{\prime}\right)=2 L_{i}^{\prime} ; \quad\left(Q_{i j} L_{i}^{\prime}\right)=R_{i j} .
\end{gathered}
$$

Theorem : The group $G$ is simple.

Suppose, indeed, an invariant subgroup $H$ of $G$ exists. Let it contain the infinitesimal transformation (not the identity),

$$
T=\sum_{i j}^{1 \ldots n} q_{i j} Q_{i j}+\sum_{i=1}^{n} l_{i}^{\prime} L_{i}^{\prime}+\sum_{i=1}^{n} l_{i} L_{i}+\sum_{i, j(i \neq i)}^{1 \ldots n}\left(r_{i j} R_{i j}+n_{i j} N_{i j}\right) \text {, }
$$

where, since $R_{i j}=R_{j i}, N_{i j}=N_{j i}$, we may take

Then must $H$ contain

$$
r_{i j}=r_{j i}, n_{i j}=n_{j i} \text {. }
$$

$$
\left(L_{k} T\right)=2 q_{k k} L_{k}+\sum_{i=1 \ldots n}^{\prime} q_{i k} N_{i k}-l_{k}^{\prime} Q_{k k}-\sum_{i=1 \ldots n}^{\prime} r_{i k} Q_{k i},
$$

$k$ being fixed and $\Sigma^{\prime}$ indicating that $i \neq k$.

Similarly must $\stackrel{i}{H}$ contain

$$
\left(L_{k}^{\prime} T\right)=-2 q_{k k} L_{k}^{\prime}-\sum_{i}^{\prime} q_{k i} R_{k i}+l_{k} Q_{k k}+\sum_{i}^{\prime} n_{i k} Q_{i k} .
$$

Hence (for $s \neq k$ ) $H$ contains

$$
\begin{gathered}
\left(L_{k}\left(L_{k} T\right)\right)=-2 l_{k}^{\prime} L_{k} ;\left(L_{k}^{\prime}\left(L_{k}^{\prime} T\right)\right)=-2 l_{k} L_{k}^{\prime} ; \\
\left(L_{s}\left(L_{k} T\right)\right)=-r_{s k} N_{s k} ;\left(L_{s}^{\prime}\left(L_{k}^{\prime} T\right)\right)=-n_{s k} R_{s k} ; \\
\left(L_{s}^{\prime}\left(L_{k} T\right)\right)=q_{s k} Q_{k s}
\end{gathered}
$$

But $\left(Q_{s k} N_{s k}\right)=-2 L_{s} ;\left(N_{s k}\left(L_{s} R_{s k}\right)\right)=-\left(N_{s k} Q_{s k}\right)=-2 L_{a}$.

Since not every $l_{k}^{\prime}, l_{k}, r_{s k}, n_{s k}, q_{s k}, q_{k k}$ is zero, $T$ not being the identity, we find that $H$ contains certainly one $L_{i}$ and by the lemma the whole of $G$.

It remains to prove that the simple group $G$ in $2 n$ variables is the homogeneous form of the largest projective group in space of $2 n-1$ dimensions (with the coördinates $\left.z, x_{1}, \cdots x_{n-1}, y_{1}, \cdots y_{n-1}\right)$ leaving invariant the linear complex defined by the Pfaff's equation :

$$
d z+\sum_{i=1}^{n-1}\left(x_{i} d y_{i}-y_{i} d x_{i}\right)=0
$$


The $n(2 n+1)$ infinitesimal transformations of the latter group are,* if $U \equiv \sum_{\nu=1}^{n-1}\left(x_{\nu} p_{\nu}+y_{\nu} q_{\nu}\right)+z r$ :

$$
\begin{gathered}
p_{i}-y_{i} r, q_{i}+x_{i} r, r, z r+U, x_{i} q_{k}+x_{k} q_{i}, \\
x_{i} p_{k}-y_{k} q_{i}, y_{i} p_{k}+y_{k} p_{i}, z p_{i}-y_{i} U, z q_{i}+x_{i} U, z U . \\
(i, k=1 \cdots n-1)
\end{gathered}
$$

If we introducet for the variables $x_{i}, y_{i}, z$ respectively $x_{i} / x_{n}, y_{i} / x_{n}, y_{n} / x_{n}$, we reach the homogeneous group $\$$ (where the new transformations are written in the same order as the old above):

$$
\begin{gathered}
Q_{i n}, R_{n i}, L_{n}^{\prime},-Q_{n n}, R_{i k} \text { and } 2 L_{i}^{\prime}, \\
Q_{k i}, N_{i k} \text { and } 2 L_{i}, N_{i n},-Q_{n i},-L_{n} \\
\left(i, k=1 \cdots n-1 ; i \neq k \text { in } N_{i k} \text { and } R_{i k}\right) .
\end{gathered}
$$

This group is evidently identical with our group $G$. By the same transformation the above Pfaff's equation takes the homogeneous form (aside from the factor $1 / x_{n}^{2}$ ):

$$
\sum_{i=1}^{n}\left(x_{i} d y_{i}-y_{i} d x_{i}\right)=0 \text {. }
$$

$\$ 4$.

Semi-simple linear homogeneous groups whose defining function§ is the sum of $n$ determinants of order $q>2$.

Of the transformations of the general linear homogeneous group in $q n$ variables, we consider those which, when operating simultaneously on $q$ independent sets of $n q$ variables, the $j^{\text {th }}$ set of which may be exhibited thus

$$
x_{i 1}^{(j)}, x_{i 2}^{(j)}, \cdots x_{i q}^{(j)}, \quad(i=1 \cdots n),
$$

leave invariant the function

$$
\varphi \equiv \sum_{i=1}^{n} D_{i}
$$

* Lre, Theorie der Transformationsgruppen, vol. II., p. 522.

† The formulæ for this change of variables are given in LIE, ibid, vol. I., p. 579.

$\ddagger$ For $n=2, i$. e., for 4 variables, this homogeneous form is given in LIE, ibid, vol. II., p. 450.

\$Discontinuous linear groups with the same defining function are mentioned (but not studied) by Jordan, l. c., p. 219. The result reached in this paragraph, where $q>2$, is wholly different from that of $\& 3$, where $q=2$, 
where $D_{i}$ denotes the determinant

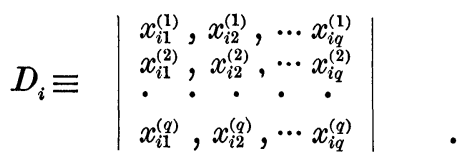

Denote by $G$ the groups of transformations thus defined and let $S$ be an arbitrary transformation of $G$; viz,

$$
\begin{gathered}
x_{i k}{ }^{\prime}=\sum_{j=1}^{n}\left(a_{j 1}^{i k} x_{j 1}+a_{j 2}^{i k} x_{j 2}+\cdots+a_{j q}^{i k} x_{j q}\right) \\
(i=1 \cdots n ; k=1 \cdots q) .
\end{gathered}
$$

The conditions that $S$ shall leave $\varphi$ invariant are :

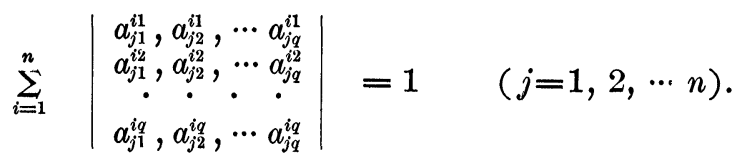

$$
\begin{aligned}
& \sum_{i=1}^{n}\left|\begin{array}{ccc}
a_{j_{1} k_{1}}^{i 1} & \cdots \cdots & a_{j_{q} k_{q}}^{i 1} \\
\vdots & & \vdots \\
a_{j_{1} k_{1}}^{i q} & \cdots \cdots & a_{j_{q} k^{k}}^{i q}
\end{array}\right|=0
\end{aligned}
$$

for $j_{s}=1 \cdots n ; k_{s}=1 \cdots q$, provided not all the $j_{s}$ 's are equal and no pair $\left(j_{\lambda}, k_{\lambda}\right)=\left(j_{\mu}, k_{\mu}\right)$.

The general infinitesimal transformation of $G$ is found by substituting in $S$

$$
a_{i k}^{i k}=1+\alpha_{i k}^{i k} \cdot \delta t, a_{r s}^{i k}=\alpha_{r s}^{i k} \cdot \delta t\left(\begin{array}{c}
i, r=1 \cdots n ; k, s=1 \cdots q \\
(r, s) \neq(i, k)
\end{array}\right)
$$

The first set of conditions (1) on the $a$ 's give at once

$$
\alpha_{j 1}^{j 1}+\alpha_{j 2}^{j 2}+\cdots+\alpha_{j q}^{j q}=0 \quad(j=1 \cdots n) .
$$

To prove that the second set (2) gives simply (for $q>2$ )

$$
\alpha_{j_{1} m}^{j l}=0\left(l, m=1 \cdots q ; j, j_{1}=1 \cdots n, j_{1}+j\right),
$$

consider first the conditions in which $j_{2}=j_{3}=\cdots=j_{q}=j$, say, and thus $j_{1} \neq j$; viz,

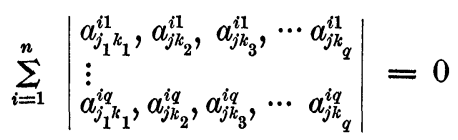

For those determinants given by $i \frac{1}{T} j$, the elements of the last $q-1$ columns all have the factor $\delta t$ and hence 
the determinants have the factor $\delta t^{q-1}$ and hence the factor $\delta t^{2}$. For the determinant $i=j$, the elements of the first column have the factor $\delta t$; likewise those of the $l^{\text {th }}$ row, if $l$ be the integer $\leqq q$ which is lacking among the numbers $k_{2}, k_{3}, \cdots k_{q}$, all different. The minor of $\alpha_{j_{1} k_{1}}^{j l}$ has the term

$$
\pm \alpha_{j k_{2}}^{j k_{2}} \alpha_{j k_{3}}^{j k_{3}} \cdots \alpha_{j k_{q}}^{j k}= \pm\left(1+\alpha_{j k_{2}}^{j k_{2}} \cdot \delta t\right) \cdots\left(1+\alpha_{j k_{q}^{q}}^{j k_{q}} \cdot \delta t\right)
$$

and but one such. Hence on expanding the determinant according to the elements of the first column, we get the term $\pm \alpha_{j_{1} k_{1}}^{j l} \cdot \delta t$ together with terms of higher order in it.

Hence $\alpha_{j_{1} k_{1}}^{j l}=0$.

The remaining ones of the conditions (2) are then satisfied identically. For unless $i=j_{s}$, the elements of the $s^{\text {th }}$ column

$$
a_{j_{s} k_{s}}^{i l}=\alpha_{j_{s} k_{s}}^{i l} \cdot \delta t=0(l=1 \cdots q) \text {. }
$$

But $i$ can not simultaneously equal $j_{1}, j_{2}, \cdots j_{q}$ for the conditions (2); hence the elements of at least one column of every determinant are all zero.

The group $G$ thus contains $n\left(q^{2}-1\right)$ linearly independent infinitesimal transformations,

$$
\delta x_{i k} \equiv x_{i k}{ }^{\prime}-x_{i k}=\alpha_{i 1}^{i k} x_{i 1}+\alpha_{i 2}^{i k} x_{i 2}+\cdots+\alpha_{i q}^{i k} x_{i q}
$$

where

$$
\sum_{k=1}^{q} \alpha_{i k}^{i k}=0 \quad(i=1 \cdots n) .
$$

The finite equations of the transformations of $G$ are then evidently

$$
x_{i k}{ }^{\prime}=\alpha_{i 1}^{i k} x_{i 1}+\cdots+\alpha_{i q}^{i k} x_{i q} \quad(i=1 \cdots n ; k=1 \cdots q),
$$

where the determinants

$$
\left|\alpha_{i m}^{i k}\right|=1 \quad(i=1 \cdots n) .
$$

Thus the group $G$ breaks up into $n$ sub-groups, those transformations in which $i$ has a fixed value forming the linear homogeneous group, leaving the determinant $D_{i}$ invariant, $i . e$. , the special linear homogeneous group in $q$ variables. Since $G$ breaks up into $n$ invariant simple sub-groups, and since all the transformations of any one are commutative with all of any other one, $G$ is a so-called half-simple (halbeinfach) group.

LEIPZIG, February 6, 1897.

[The contents of $\S 3$ and $\S 4$ were presented February 19 th before the Groups-Seminar of Professor Lie, who stated that the interesting result of $\$ 4$ was new and not what one would have expected.] 